\title{
Síntese e avaliação de nanopartículas de sílica mesoporosas na liberação controlada de feromônios repelentes de abelha
}

\section{(Synthesis and evaluation of mesoporous silica nanoparticles in the controlled release of bee repellent pheromones)}

\author{
E. T. Zanoni ${ }^{1 *}$, G. D. Savi ${ }^{1}$, M. F. Valadares ${ }^{2}$, E. Angioletto ${ }^{1}$ \\ ${ }^{1}$ Universidade do Extremo Sul Catarinense, Programa de Pós-Graduação em Ciências e Engenharia de \\ Materiais, Av. Universitária 1106, 88806-000, Criciúma, SC, Brasil \\ ${ }^{2}$ Universidade do Estado de Santa Catarina, 89219-710, Joinville, SC, Brasil
}

\begin{abstract}
Resumo
Neste estudo foi avaliado o armazenamento e liberação de feromônios repelentes de abelhas em uma nanoestrutura de sílica mesoporosa. O nanomaterial foi sintetizado via método sol-gel e caracterizado por microscopia eletrônica de transmissão, espectroscopia de infravermelho e método BET. As nanopartículas mesoporosas apresentaram-se com área superficial de $1084 \mathrm{~m}^{2} / \mathrm{g}$ exercendo a função desejada para a proposta do estudo. Os feromônios foram carregados nos percentuais de $10 \%, 20 \%$ e $40 \%$ e a efetividade dos carregamentos foi analisada por termogravimetria. Em seguida foi avaliado o comportamento de liberação do nanossistema, sendo possível observar que as nanopartículas influenciaram de forma significativa no perfil de liberação dos feromônios, retardando a sua taxa de liberação. Dos ensaios de avaliação do comportamento de liberação concluiu-se que as nanopartículas mesoporosas prolongaram a atividade de liberação dos feromônios permanecendo com essa função por vários dias.
\end{abstract}

Palavras-chaves: nanopartículas, sílica mesoporosa, repelente, abelhas, liberação controlada.

\begin{abstract}
This study evaluated the storage and release of repellent pheromones of bees in a mesoporous silica nanostructure. The nanomaterial was synthesized by sol-gel method and characterized by transmission electron microscopy, infrared spectroscopy, and BET method. The mesoporous nanoparticles presented a surface area of $1084 \mathrm{~m}^{2} / \mathrm{g}$, exerting the desired function for the study proposal. The pheromones were loaded in percentages of $10 \%, 20 \%$, and $40 \%$ and the effectiveness of the loads was analyzed by thermogravimetry. Next, the nanosystem release behavior was evaluated, and it was possible to observe that the nanoparticles had a significant influence on the release profile of the pheromones, delaying their release rate. From the evaluation of the release behavior, it was concluded that the mesoporous nanoparticles prolonged the activity of releasing the pheromones remaining with this function for several days.
\end{abstract}

Keywords: nanoparticles, mesoporous silica, repellent, bees, controlled release.

\section{INTRODUÇÃO}

A nanotecnologia tem sido um atrativo nos últimos tempos pelas suas aplicações que variam desde aplicações farmacêuticas [1, 2], catálise e geração de energia [3-5], semicondutores para circuitos eletrônicos em miniatura, biossensores [6, 7], entre tantas outras aplicações. Recentemente a nanotecnologia vem ganhando mais espaço na área agrícola, atuando como nanofertilizantes e nano-herbicidas diminuindo a porção de agentes químicos que causam danos ao meio ambiente [8]. Nanomateriais funcionalizados apresentam aplicações no combate às

*elton.tz@hotmail.com

(D) https://orcid.org/0000-0003-3117-7178 pragas atuando como inseticidas e/ou pesticidas, auxiliando e promovendo melhores produções de alimentos causando impactos positivos na economia [9-14]. Em alguns cultivos, como o do maracujá e da uva, as abelhas são consideradas por agricultores uma espécie de praga, que eventualmente ocasionam a destruição parcial ou total do fruto durante a busca por alimentos. Para evitar que haja dano no cultivo, frequentemente os agricultores aplicam pesticidas, que em decorrência de sua atividade ocasionam a morte das abelhas com graves efeitos ao meio ambiente [15-18]. Na última década a preocupação com as abelhas ficou mais intensa após o desaparecimento dessas em muitos lugares do planeta, fenômeno esse conhecido como distúrbio do colapso das colônias (DCC), onde colônias inteiras desapareceram em decorrência de diversos fatores, entre elas o uso de 
alguns pesticidas e agrotóxicos nas lavouras [19]. Uma das formas usadas para o gerenciamento de pragas é o uso de feromônios [20], que podem atuar no monitoramento de pestes e em armadilhas ou repelindo. O termo 'feromônio' foi proposto por Karlson e Lüscher, derivado do grego pherin (transferir) e hormon (excitar), e significa substâncias que são secretadas para fora por um indivíduo e recebido por outro causando uma reação específica [21]. Para abelhas o acetato de octila e a 2-heptanona, mostrados nas Figs. 1a e $1 b$, respectivamente, possuem ação repelente $[18,22]$ e por se tratar de substâncias feromônias, ou seja, produzidas pelo próprio inseto agindo no comportamento alertando-as em situações de perigo, podem ser usadas para função desejada com pouco dano aos mesmos [23-26]. a)

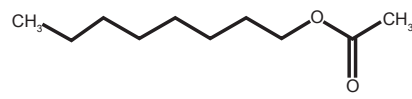

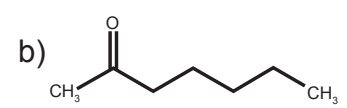

Figura 1: Estrutura molecular do feromônio n-acetato de octila (a) e 2-heptanona (b).

[Figure 1: Molecular structure of pheromone octyl n-acetate (a) and 2-heptanone $(b)$.]

Nanopartículas de sílica são consideradas nanoreservatórios robustos e estáveis e em especial as mesoporosas por possuírem poros com diâmetro entre $2 \mathrm{e}$ $50 \mathrm{~nm}$ [27] possuem flexibilidade na adsorção de moléculas com tamanho e geometria variada [28], são biocompatíveis, não tóxicas, facilmente sintetizadas e com mesoporosidade estável [29] e por isso esse nanomaterial tem sido cada vez mais usado em sistemas de carreamento controlado. Os nanomateriais vêm com o intuito de melhorar a eficácia na aplicação. Repelentes para abelhas têm sido registrados em patentes e descritos na literatura [15, 30], porém com o uso da nanotecnologia os princípios ativos repelentes podem ter seu tempo de efeito prolongado e um uso menor de sua porção. $\mathrm{O}$ aumento da eficiência nas propriedades decorre das características de adsorção química e física que os nanomateriais apresentam. Este estudo teve como objetivo sintetizar e caracterizar as nanopartículas de sílica mesoporosas (MSN), bem como avaliar o carregamento e a liberação controlada dos feromônios acetato de octila e 2-heptanona carregados nas MSN.

\section{MATERIAIS E MÉTODOS}

Reagentes: para o estudo deste trabalho foram utilizados os seguintes reagentes: ortossilicato de tetraetila (TEOS), acetato de octila e 2-heptanona da Sigma Aldrich (Estados Unidos), etanol absoluto da Synth (Brasil), hidróxido de amônio da Química Moderna (Brasil) e brometo de cetiltrimetilamônio (CTAB) da J.T. Baker (Estados Unidos).

Síntese das nanopartículas de sílica mesoporosas: as MSN foram preparadas pelo método sol-gel, que consistiu na hidrolização do TEOS catalisado por uma base. Foram dissolvidos $533 \mathrm{mg}$ de CTAB em 293,5 mL de uma solução 0,5 $\mathrm{M}$ de $\mathrm{NH}_{4} \mathrm{OH}$ sob agitação magnética. O sistema foi aquecido até $50{ }^{\circ} \mathrm{C}$. Em seguida, foram adicionados gota a gota $12,5 \mathrm{~mL}$ de uma solução $0,2 \mathrm{M}$ de TEOS dissolvido em etanol absoluto seguido de $48,5 \mathrm{~mL}$ de etanol absoluto. A mistura permaneceu sob agitação magnética a $50{ }^{\circ} \mathrm{C}$ por $5 \mathrm{~h}$ e então acrescentaram-se mais $12,5 \mathrm{~mL}$ da solução de TEOS e $48,5 \mathrm{~mL}$ de etanol absoluto, permanecendo em agitação a $50^{\circ} \mathrm{C}$ por mais $1 \mathrm{~h}$. Após esse período, o material foi centrifugado e lavado com água e etanol diversas vezes e seco em estufa na temperatura média de $80{ }^{\circ} \mathrm{C}$. Para a remoção do surfactante foi utilizada uma solução $0,1 \mathrm{M}$ de $\mathrm{HCl}$ em etanol, na qual o nanomaterial foi dispersado permanecendo em agitação magnética a $40{ }^{\circ} \mathrm{C}$ por $2 \mathrm{~h}$. Após essa etapa o nanomaterial foi lavado diversas vezes com etanol e seco na estufa [31].

Carregamento dos princípios ativos: o carregamento consistiu em dispersar $250 \mathrm{mg}$ de MSN em $25 \mathrm{~mL}$ de solução com 10\%, $20 \%$ ou $40 \%$ em volume do repelente em etanol. A mistura permaneceu em agitação magnética a $60{ }^{\circ} \mathrm{C}$ por $24 \mathrm{~h}$ para a completa adsorção. Após este período o material foi centrifugado e seco em baixa temperatura para eliminação do solvente remanescente. Quando seco, o material foi armazenado em baixa temperatura para evitar a liberação prematura do feromônio.

Caracterização: as MSN foram caracterizadas por espectroscopia de infravermelho (FT-IR) usando pastilhas de $\mathrm{KBr}$ (proporção 1:100 massa/massa do material em relação ao $\mathrm{KBr}$ ) com auxílio de um equipamento Shimadzu, IRPrestige, objetivando a avaliação da formação de $\mathrm{SiO}_{2} \mathrm{e}$ a confirmação da presença dos princípios ativos adsorvidos na matriz de sílica. A morfologia das nanopartículas foi avaliada por microscopia eletrônica de transmissão (MET) em um equipamento Jeol, JEM 2100, operado com voltagem de aceleração de $200 \mathrm{kV}$. A área de superfície foi determinada pelo método Brunauer-Emmett-Teller (BET) e a distribuição de poros usando isotermas de adsorção-dessorção de $\mathrm{N}_{2}$ aplicando o método Barret-Joyner-Halenda (BJH) em um equipamento Quantachrome, NOVA-1200e. Para investigar o percentual de feromônio contido na nanoestrutura e o comportamento térmico das amostras foram efetuadas análises térmicas em um equipamento TA, Q600, com termogravimetria e calorimetria diferencial de varredura acoplada (TGA/DSC), na faixa de 25 a $600{ }^{\circ} \mathrm{C}$ com taxa de aquecimento de $5^{\circ} \mathrm{C} / \mathrm{min}$ em atmosfera de nitrogênio.

Teste de liberação dos feromônios: para avaliar o perfil de liberação da nanoestrutura previamente carregada com os feromônios nos percentuais de 10\%, $20 \%$ e $40 \%$, foi usado uma ultramicrobalança da PerkinElmer, AD-8000, com resolução de $0,1 \mu \mathrm{g}$ com temperatura controlada em $22,0{ }^{\circ} \mathrm{C}$ com variação máxima de $\pm 0,9{ }^{\circ} \mathrm{C}$. Para avaliação da liberação do feromônio depositado diretamente sobre as MSN, foi utilizada uma quantidade de massa fixa de $20 \mathrm{mg}$ do nanomaterial em um recipiente de polímero revestido com papel alumínio, na qual alíquotas de 20 e $50 \mu \mathrm{L}$ do feromônio 2-heptanona foram diretamente depositadas. A liberação do feromônio foi monitorada em uma balança analítica da Bioprecisa, FA-2104N, com resolução de $0,1 \mathrm{mg}$ em temperatura ambiente de $22{ }^{\circ} \mathrm{C}$. 


\section{RESULTADOS E DISCUSSÃO}

Formação e composição das MSN: a formação de nanopartículas de sílica ocorreu em três etapas denominadas hidrólise, condensação de álcool e condensação de água, cujas reações foram, respectivamente [32]:

$$
\begin{aligned}
& \mathrm{Si}-\left[\mathrm{OC}_{2} \mathrm{H}_{5}\right]_{4}+4 \mathrm{H}_{2} \mathrm{O} \rightarrow \mathrm{Si}-(\mathrm{OH})_{4}+4 \mathrm{C}_{2} \mathrm{H}_{5} \mathrm{OH} \\
& \mathrm{Si}-(\mathrm{OH})_{4}+\mathrm{Si}-\left[\mathrm{OC}_{2} \mathrm{H}_{5}\right]_{4} \rightarrow \equiv \mathrm{Si}-\mathrm{O}-\mathrm{Si} \equiv+4 \mathrm{C}_{2} \mathrm{H}_{5} \mathrm{OH}(\mathrm{A}) \\
& \mathrm{Si}-(\mathrm{OH})_{4}+\mathrm{Si}-(\mathrm{OH})_{4} \rightarrow \equiv \mathrm{Si}-\mathrm{O}-\mathrm{Si} \equiv+4 \mathrm{H}_{2} \mathrm{O}
\end{aligned}
$$

A formação das MSN ocorreu de forma similar ao método descrito por Stöber [33], exceto pela presença do surfactante que serviu para a formação dos mesoporos. As moléculas do CTAB formam micelas ocorrendo a policondensação durante a hidrólise do TEOS, formando mesoporos na nanopartícula de sílica. $\mathrm{O}$ tipo de surfactante altera significativamente o formato dos mesoporos; neste estudo o CTAB formou um tipo específico de MSN denominado MCM-41, na qual os mesoporos tendem a um arranjo hexagonal.

Caracterização das MSN: o espectro deFT-IR apresentado na Fig. 2 mostra as bandas de vibração características de sílica, em $3451 \mathrm{~cm}^{-1}$ associada ao grupo -OH, que pode ser decorrente da ligação $\mathrm{H}-\mathrm{OH}$ ou do grupo silanol Si$\mathrm{OH}$. Em $1640 \mathrm{~cm}^{-1}$ são vibrações de deformação angular do -OH de moléculas de água adsorvidas na superfície da nanopartícula e em $1089 \mathrm{~cm}^{-1}$ é o estiramento assimétrico de Si-O-Si. As vibrações em 956 e $792 \mathrm{~cm}^{-1}$ são deformações assimétricas fora do plano de grupo silanol e por fim em 454 $\mathrm{cm}^{-1}$ são vibrações associadas à deformação angular de $\mathrm{Si}$ O-Si [34-36]. As micrografias de MET (Fig. 3) confirmaram que as partículas de sílica foram da ordem nanométrica, mesoporosas e não esféricas, possuindo formato lamelar em forma de disco e apresentaram boa dispersão. Os mesoporos não apresentaram uniformidade caracterizando-os como desordenados e parcialmente um arranjo do tipo wormhole [37]. A área superficial analisada por BET resultou em um valor de $1084 \mathrm{~m}^{2} / \mathrm{g}$. A Fig. 4 mostra a isoterma de adsorção

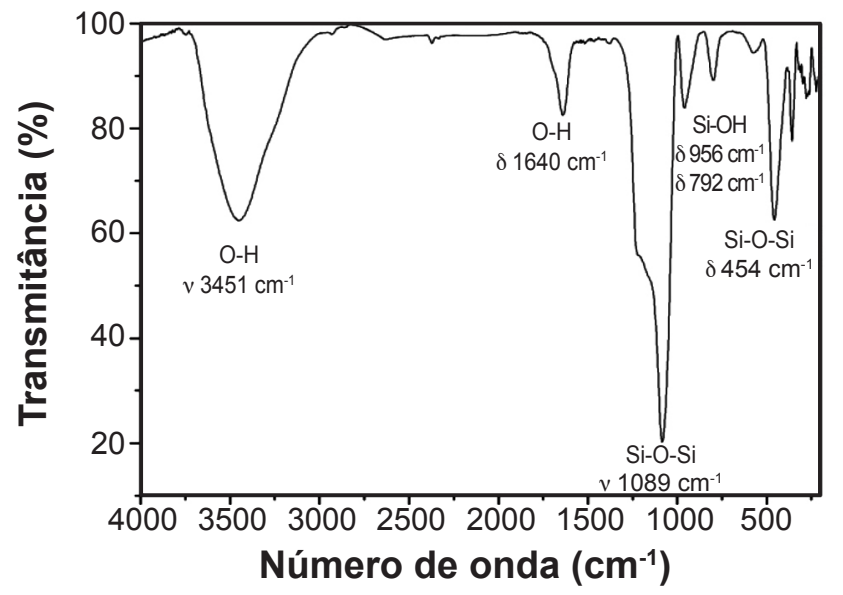

Figura 2: Espectro de FTIR de MSN.

[Figure 2: FTIR spectrum of MSN.]

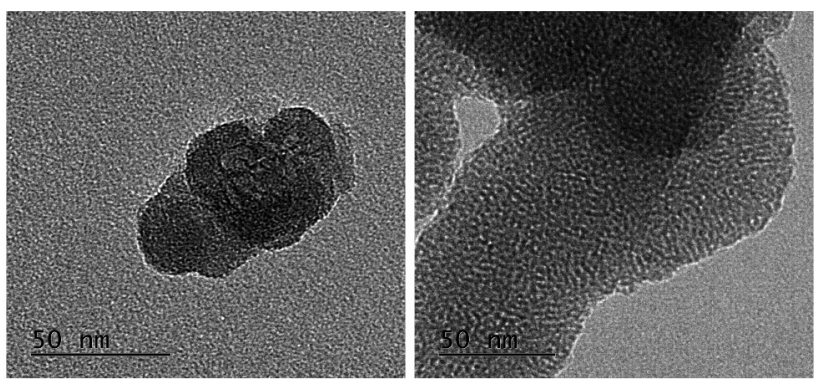

Figura 3: Micrografias eletrônicas de transmissão das nanopartículas de sílica mostrando a mesoporosidade.

[Figure 3: Transmission electron micrographs of silica nanoparticles showing mesoporosity.]

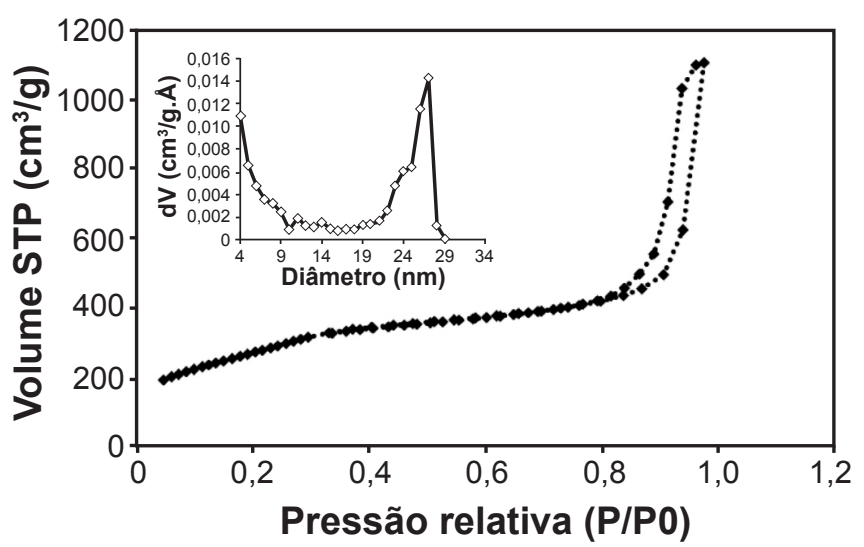

Figura 4: Isoterma de sorção de $\mathrm{N}_{2}$ de MSN.

[Figure 4: $N_{2}$ sorption isotherm of MSN.]

e dessorção de $\mathrm{N}_{2}$ a qual mostra um típico perfil de isoterma do tipo IV com histerese que é um comportamento associado à condensação capilar em mesoporos. Além disso, a forma das alças de histerese é classificada no tipo H3, característica de poros na forma de fendas $[38,39]$, que no perfil de distribuição de poros tem um tamanho de 4 e $27 \mathrm{~nm}$, sendo este último o que mais prevaleceu na amostra.

Avaliação do carregamento dos feromônios: na Fig. 5 estão dispostos os espectros de FTIR dos feromônios carregados. $\mathrm{O}$ acetato de octila (Fig. 5a) possuiu bandas de vibração características na região de $2931 \mathrm{~cm}^{-1}$ associadas ao estiramento assimétrico da ligação $\mathrm{C}-\mathrm{H}$ da cadeia alquílica, em $1748 \mathrm{~cm}^{-1}$ vibração de estiramento da ligação $\mathrm{C}=\mathrm{O}$ e em $1235 \mathrm{~cm}^{-1}$ do $\mathrm{C}-\mathrm{O}$ do grupo éster. No espectro da MSN/acetato de octila apareceram tanto as bandas características da sílica como também do feromônio, confirmando o carregamento. Com o aumento do percentual, a característica da vibração da região de $3500 \mathrm{~cm}^{-1}$ alterou, apresentando bandas definidas em 3566 e $3413 \mathrm{~cm}^{-1}$. Essa alteração pode ter sido ocasionada pela interação da hidroxila presente no grupo silanol ( $\mathrm{Si}-\mathrm{OH})$ com a molécula de feromônio adsorvida na superfície da nanopartícula. Para os carregamentos de 2-heptanona, os espectros da Fig. $5 \mathrm{~b}$ evidenciaram sua presença pelas vibrações na região $2931 \mathrm{~cm}^{-1}$ e na mudança da característica da vibração em $3500 \mathrm{~cm}^{-1}$, que apresentou duas bandas de vibração definidas em 3557 e $3413 \mathrm{~cm}^{-1} \mathrm{em}$ alto percentual do feromônio, como também foi observado para o acetato de octila. 

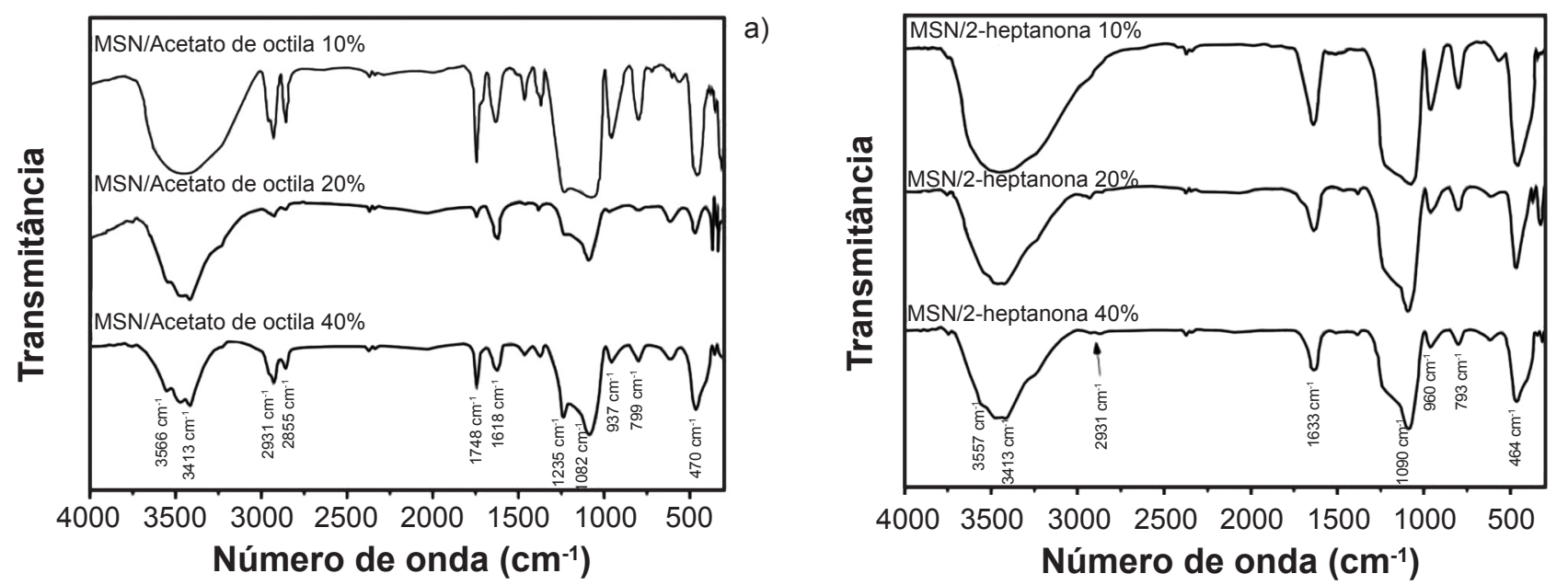

b)

Figura 5: Espectros de FTIR dos feromônios acetato de octila (a) e 2-heptanona (b) carregados em MSN.

[Figure 5: FTIR of the pheromones octyl acetate (a) and 2-heptanone (b) loaded on MSN.]
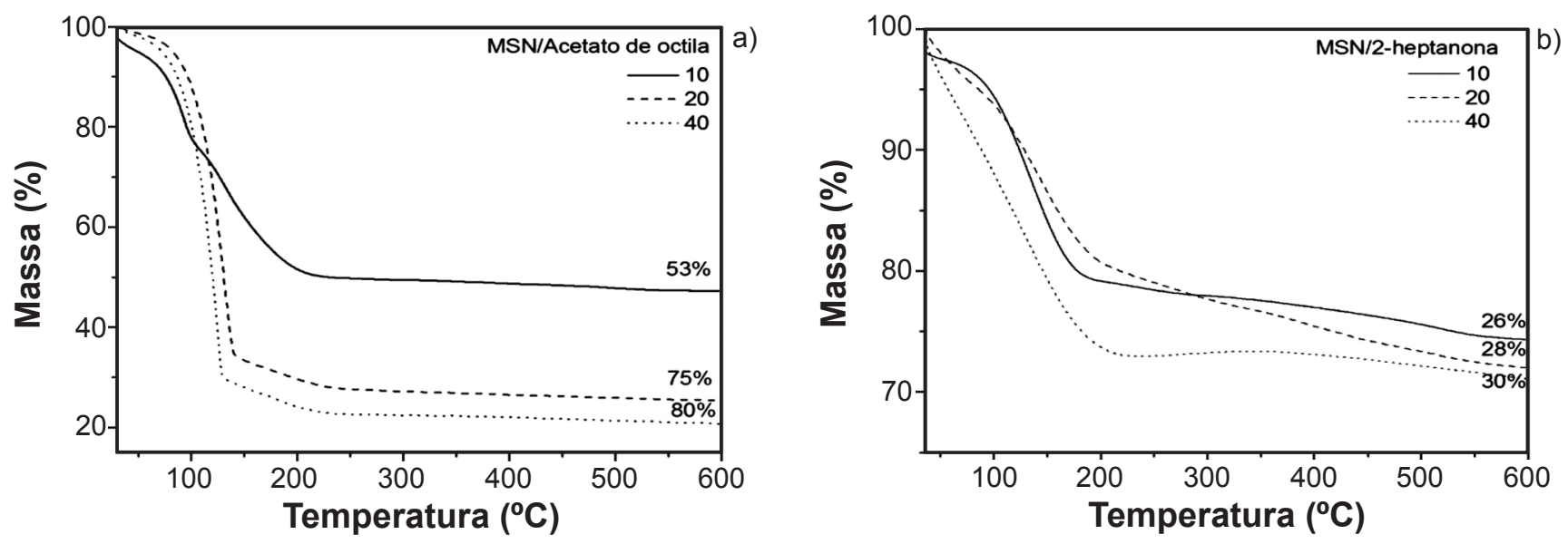

Figura 6: Curvas de TGA das amostras de MSN carregadas com feromônios acetato de octila (a) e 2-heptanona (b).

[Figure 6: TGA curves of MSN samples loaded with pheromones octyl acetate (a) and 2-heptanone (b).]
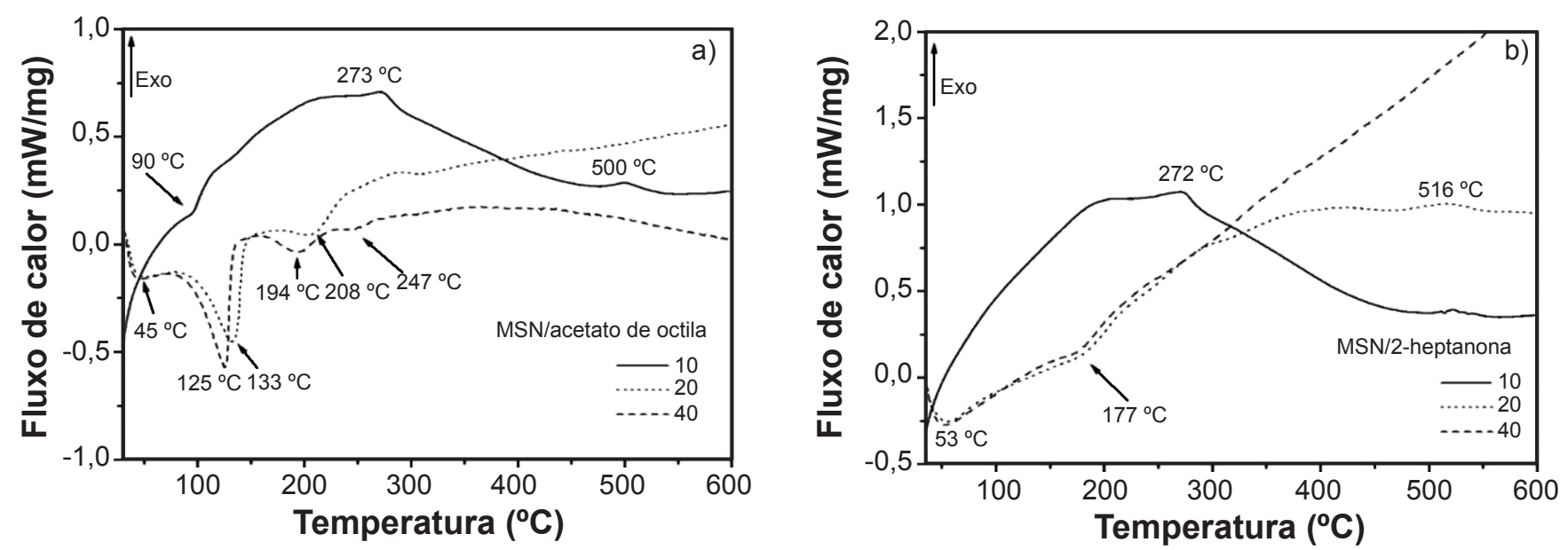

Figura 7: Curvas de DSC das amostras de MSN carregadas com feromônios acetato de octila (a) e 2-heptanona (b).

[Figure 7: DSC curves of MSN samples loaded with pheromones octyl acetate (a) and 2-heptanone (b).]

Os termogramas da Fig. 6 confirmaram a presença e a quantidade de feromônio carregadas na estrutura mesoporosa. $\mathrm{O}$ acetato de octila teve um carregamento mais eficiente que a 2-heptanona e isso ocorreu pelas características moleculares de cada feromônio. $\mathrm{O}$ acetato é um éster possuindo um grupo mais polar sendo menos volátil e é fortemente adsorvido na superfície da nanopartícula mesoporosa. A carga máxima de feromônio que a amostra de MSN/acetato de octila 

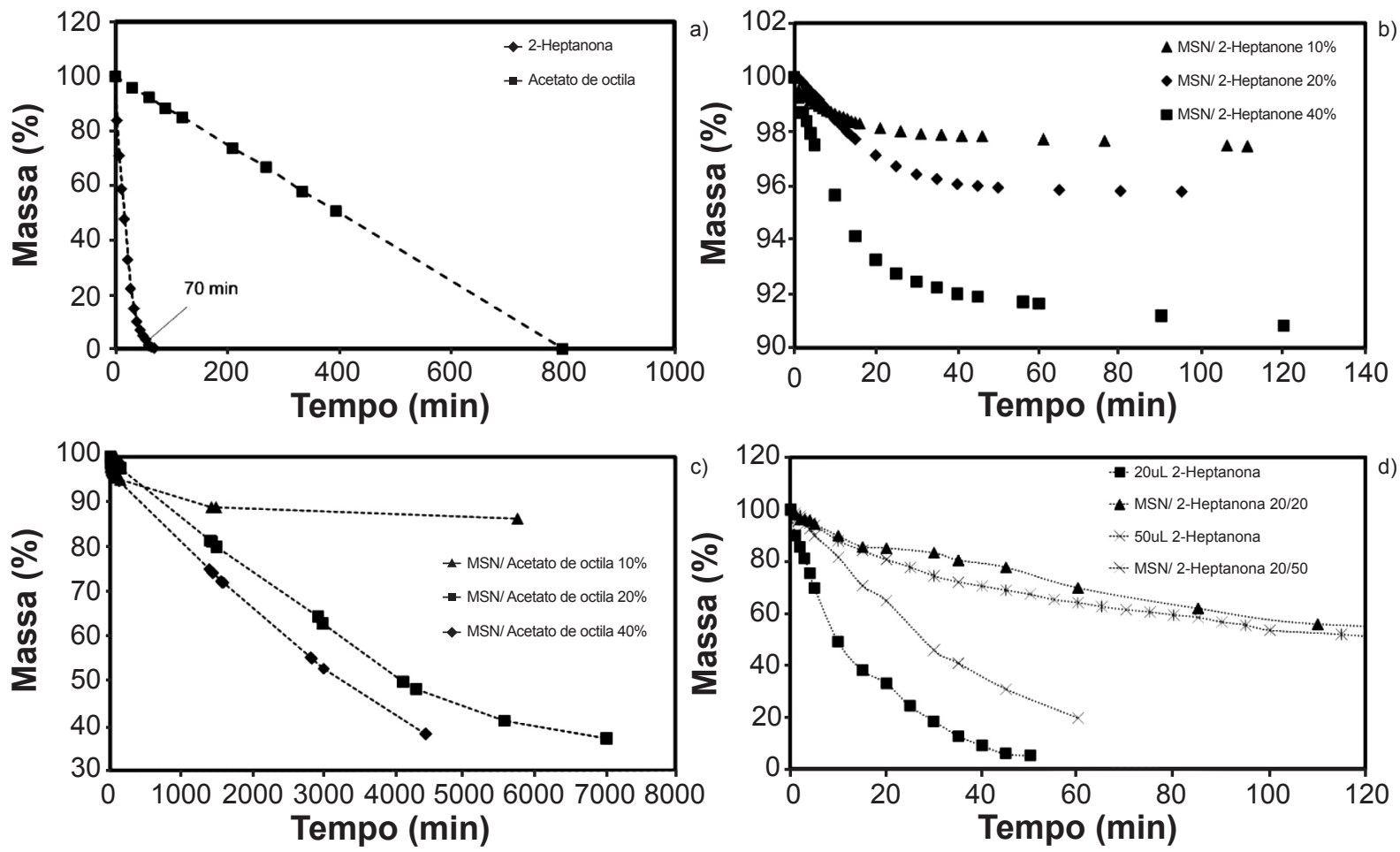

Figura 8: Comportamento do perfil de liberação dos feromônios: a) volatilidade dos feromônios puros; b) perfil de liberação da heptanona carregada em MSN; c) perfil de liberação de acetato de octila carregado em MSN; e d) perfil de liberação da 2-heptanona pelo método 2.

[Figure 8: Behavior of the pheromone release profile: a) volatility of pure pheromones b) heptanone release profile loaded on MSN; c) release profile of octyl acetate loaded in MSN; and d) 2-heptanone release profile by method 2.]

atingiu foi $80 \%$. As amostras com 2-heptanona atingiram um máximo de $30 \%$, ocasionado pela alta volatilidade por possuir um grupo menos polar (cetona) que não ancorou de forma tão eficiente na nanoestrutura. As curvas de DSC da Fig. 7, além de confirmar que o acetato necessita de mais energia para sua liberação da mesoestrutura, indicaram uma liberação em multicamada com três picos endotérmicos a 126, 194 e $208{ }^{\circ} \mathrm{C}$ para a amostra com maior quantidade de carregamento. As moléculas mais próximas da superfície foram liberadas em maior quantidade, apresentando maior perda observada no termograma da Fig. 6 , enquanto que as moléculas localizadas mais internamente foram liberadas em $194{ }^{\circ} \mathrm{C}$. Em $247{ }^{\circ} \mathrm{C}$ a hipótese é que sejam moléculas adsorvidas em cavidades microporosas. A 2-heptanona por ser mais volátil absorve menos energia em sua liberação, apresentando um pico mais tênue em $177^{\circ} \mathrm{C}$ para os dois carregamentos de maior percentual. Os picos exotérmicos que surgiram nas duas amostras podem estar associados a um processo de cristalização e rearranjo dos mesoporos.

Impacto das MSN na taxa de liberação do feromônio: cada um dos feromônios estudados neste trabalho possuiu uma taxa natural de liberação (Fig. 8a). Para o acetato de octila foi encontrada uma taxa de $672 \mu \mathrm{g} / \mathrm{h}$ e para a 2-heptanona uma taxa de $17490 \mu \mathrm{g} / \mathrm{h}$. A taxa de liberação dos feromônios carregados na nanoestrutura sofreu alterações pela ação da nanoestrutura, diminuindo consideravelmente, como pode ser observado nas Figs. 8b, 8c e 8d. A Fig. 8b mostra o comportamento de liberação da 2-heptanona na nanopartícula mesoporosa durante $120 \mathrm{~min}$; observou-se que houve uma liberação mais rápida nos primeiros minutos mudando o perfil de dessorção após 40 min, adotando uma cinética mais lenta. Para o percentual maior de carregamento, de 0 a 20 min a taxa de liberação constatada foi de $912 \mu \mathrm{g} / \mathrm{h}$, associada às moléculas que se encontraram na região imediata à superfície da nanopartícula. Após 40 min a taxa mudou para $36 \mu \mathrm{g} / \mathrm{h}$, associada às moléculas que se encontraram localizadas nas cavidades mais internas da nanopartícula. Inicialmente a taxa foi $\sim 20$ vezes menor e posteriormente diminuiu até atingir $\sim 485$ vezes menos, quando comparada com a taxa do feromônio puro. A Fig. 8c mostra a liberação do feromônio acetato de octila ancorado na nanoestrutura onde se nota que a liberação do feromônio ocorreu por um longo período de tempo, atingindo percentuais de liberação distintos dependentes da quantidade de feromônio contido na amostra. Foi observado que até 4500 min o nanorrepelente na condição de maior carregamento liberou $75 \%$ dos $80 \%$ encontrados no termograma.

A 2-heptanona por ser mais volátil foi usada como modelo para cinética de curta duração (Fig. 8d). Duas alíquotas $(20$ e $50 \mu \mathrm{L})$ do feromônio foram adicionadas sobre uma massa de $20 \mathrm{mg}$ de MSN e avaliada sua taxa de liberação comparada com a volatilidade natural. Observou-se que o retardo da liberação com a presença das nanopartículas ocorreu de forma significativa. A taxa do feromônio puro na quantidade de $20 \mu \mathrm{L}$ inicialmente foi de $0,778 \mathrm{mg} / \mathrm{min}$ e depois de alguns minutos mudou para $0,157 \mathrm{mg} / \mathrm{min}$. Com 
a nanopartícula inicialmente a taxa foi $0,197 \mathrm{mg} / \mathrm{min}$, tendo a mudança da taxa depois de $85 \mathrm{~min}$ para $0,021 \mathrm{mg} / \mathrm{min}$. Comparativamente, as nanopartículas retardaram a taxa nos primeiros 5 min em 4 vezes e 7,5 vezes para a segunda etapa da liberação. Para a amostra com $50 \mu \mathrm{L}$ de feromônio a taxa inicial sem a nanopartícula foi de $0,714 \mathrm{mg} / \mathrm{min}$ e de $0,426 \mathrm{mg} / \mathrm{min}$ depois de $15 \mathrm{~min}$. Os $50 \mu \mathrm{L}$ do feromônio depositados no nanomaterial teve nos primeiros $15 \mathrm{~min}$ uma taxa de liberação de $0,430 \mathrm{mg} / \mathrm{min}$ e após $95 \mathrm{~min}$ uma taxa de $0,053 \mathrm{mg} / \mathrm{min}$. Comparando as taxas de liberação do feromônio puro e carregado nesta condição foi encontrada uma diferença de 1,6 e 8,0 vezes, respectivamente. Isso demonstrou que a taxa de liberação depende da quantidade de feromônio que está carregada na nanoestrutura e que as nanopartículas mesoporosas atuaram de forma significativa na cinética de difusão do feromônio, podendo prolongar em até 8 vezes. Os resultados corroboraram a literatura onde foram empregados materiais porosos para retardar a liberação de feromônios e que materiais com elevada área de superfície promovem alteração na liberação da substância volátil. Em casos dos mesoporos a temperatura de volatilização da substância carregada pode exceder a sua temperatura de ebulição devido ao fenômeno de condensação capilar [40]. Outros estudos com feromônios usando nanogéis também resultaram em um prolongamento na liberação desses voláteis, podendo assim ser empregados no controle de pragas por vários dias em uma única aplicação [41].

\section{CONCLUSÕES}

As nanopartículas de sílica sintetizadas resultaram em uma nanoestrutura mesoporosa amorfa com área de superfície elevada tornando-as adequadas para a função de armazenar os feromônios repelentes de abelhas avaliadas neste trabalho. A eficiência do carregamento dos feromônios variou de acordo com a característica molecular do feromônio, onde se obteve resultados melhores com o acetato de octila, que por ser uma molécula mais polar que a 2-heptanona foi mais favorável para a adsorção realizando interação com os grupos silanol presentes na superfície da nanopartícula mesoporosa. A liberação dos feromônios foi sensível às características de volatilidade do mesmo e à carga contida na nanoestrutura, sendo que o perfil de liberação possuiu estágios indicando adsorção das moléculas em multicamadas. A cinética de liberação avaliadas na nanoestrutura confirmou que houve um retardo significante podendo diminuir a taxa de liberação fazendo com que o sistema possa durar por dias dependendo da carga aplicada no nanomaterial.

\section{AGRADECIMENTOS}

Os autores agradecem a CAPES, CNPq e às instituições UNESC e UDESC pelo suporte prestado.

\section{REFERÊNCIAS}

[1] E. Cardoso, G.T. Rezin, E.T. Zanoni, F. De Souza
Notoya, D. Dimer Leffa, A.P. Damiani, F. Daumann, J.C.O. Rodriguez, R. Benavides, L. da Silva, V. Andrade, M.M.S. Paula, Mutat. Res. 766-767 (2014) 25.

[2] M.B. Dohnert, G.K.Ferreira, P.C.L. Silveira, E.T.Zanoni, L.H. Dohnert, C.T. de Souza, M.M.S. Paula, Inflammation 38 (2014) 1044.

[3] S. Yan, L. Gao, S. Zhang, W. Zhang, Y. Li, L. Gao, Electrochim. Acta 94 (2013) 159.

[4] K.W. Park, J.H. Choi, S.A. Lee, C. Pak, H. Chang, Y.E. Sung, J. Catal. 224 (2004) 236.

[5] A. Wieckowski, E.R. Savinova, C.G. Vayenas, Catalysis and electrocatalysis at nanoparticle surfaces, Taylor Francis, New York (2004) 970.

[6] Z. Bagheryan, A. Noori, S. Zahra Bathaie, M. YousefElahi, M.F. Mousavi, Biosens. Bioelectron. 77 (2016) 767.

[7] M.I.H. Ansari, S. Hassan, A. Qurashi, F.A. Khanday, Biosens. Bioelectron. 85 (2016) 247.

[8] M.A. Ali, I. Rehman, A. Iqbal, S. Din, A.Q. Rao, A. Latif, T.R. Samiullah, S. Azam, T. Husnain, Adv. Life Sci. 1 (2014) 129.

[9] C. Parisi, M. Vigani, E. Rodríguez-Cerezo, Nano Today 10 (2015) 124.

[10] M. Kah, T. Hofmann, Environ. Int. 63 (2014) 224.

[11] Z.-Z. Li, S.-A. Xu, L.-X. Wen, F. Liu, A.-Q. Liu, Q. Wang, H.Y. Sun, W. Yu, J.F. Chen, J. Control. Release 111 (2006) 81.

[12] H.T. Gul, S. Saeed, F. Zafar, A. Khan, S.A. Manzoor, Appl. Sci. Bus. Econ. 1 (2014) 23.

[13] N. Debnath, S. Mitra, S. Das, A. Goswami, Powder Technol. 221 (2012) 252.

[14] H.J. Park, S.H. Kim, H.J. Kim, S.H. Choi, Plant Pathol. J. 22 (2006) 295.

[15] B.M. Sacking, Y. Fishman, "Honey bee repellent composition comprising tea tree oil", U.S. Pat. 5783863 (1998).

[16] M.T. Sanford, "Protecting honey bees from pesticides", CIR534, Un. Florida (2011).

[17] D.T.M. Souza, A. Charlier, M.M. Rossi, A.D.S. Pinto, R.H. Nogueira-Couto, Acta Sci. Anim. Sci. 25 (2003) 1.

[18] D.T. Malerbo-Souza, R.H. Nogueira-Couto, Braz. Arch. Biol. Technol. 47 (2004) 121.

[19] J.S. Pettis, E.M. Lichtenberg, M. Andree, J. Stitzinger, R. Rose, D. VanEngelsdorp, PLoS One 8 (2013) e70182.

[20] I. Yosha, A. Shani, S. Magdassi, J. Agric. Food Chem. 56 (2008) 8045.

[21] J.Y. Yew, H. Chung, Prog. Lipid Res. 59 (2015) 88.

[22] J.P. Rieth, W.T. Wilson, M.D. Levin, J. Apic. Res. 25 (1986) 78.

[23] J. Free, A. Ferguson, J. Simpkins, B. Al-Sa'ad, J. Apic. Res. 22, 4 (1983) 214.

[24] J.B. Free, Anim. Behav. 9 (1961) 193.

[25] M.S. Blum, H.M. Fales, K.W. Tucker, A.M. Collins, J. Apic. Res. 17 (1978) 218.

[26] D.T. Malerbo-Souza, R.H. Nogueira-Couto, Sci. Agric. 55 (1998) 1.

[27] U. Ciesla, F. Schüth, Micro. Meso. Mater. 27 (1999) 131. 
[28] F. Hoffmann, M. Cornelius, J. Morell, M. Fröba, Angew. Chem. Int. Ed. 45 (2006) 3216.

[29] M. Llinàs, D. Sánchez-García, Afinidad 71, 565 (2014) 20.

[30] D.G. Naik, H. Vaidya-Kannur, P.V. Deshpande, C.N. Dandge, G.V.P. Reddy, Ann. Entomol. Soc. Am. 108 (2014) 18.

[31] Y. Lin, C. Tsai, H. Huang, C. Kuo, Y. Hung, D. Huang, Y. Chen, C. Mou, Chem. Mater. 17 (2005) 4570.

[32] G.Y. Bae, B.G. Min, Y.G. Jeong, S.C. Lee, J.H. Jang, G.H. Koo, J. Colloid Interf. Sci. 337 (2009) 170.

[33] W. Stöber, A. Fink, E. Bohn, J. Colloid Interf. Sci. 26 (1968) 62.

[34] S. Musić, N. Filipović-Vinceković, L. Sekovanić, Braz. J. Chem. Eng. 28 (2011) 89.

[35] A. Mourhly, M. Khachani, A. El Hamidi, M. Kacimi,
M. Halim, S. Arsalane, Nanomater. Nanotechnol. 5 (2015) 1 .

[36] A. Alessi, S. Agnello, G. Buscarino, F.M.M. Gelardi, J. Non-Cryst. Solids 362 (2013) 20.

[37] K. Möller, J. Kobler, T. Bein, Adv. Funct. Mater. 17 (2007) 605.

[38] Y. Zhang, W. Zhu, Q. Lin, J. Han, L. Jiang, L. Zhang, Int. J. Nanomed. 10 (2015) 3291.

[39] D.A.H.-D. Río, A.F. Aguilera-Alvarado, I. CanoAguilera, M. Martínez-Rosales, S. Holmes, Mater. Sci. Appl. 3 (2012) 485.

[40] A.J. Stipanovic, P.J. Hennessy, F.X. Webster, Y. Takahashi, J. Agric. Food Chem. 52 (2004) 2301.

[41] D. Bhagat, S.K. Samanta, S. Bhattacharya, Sci. Rep. 3 (2013) 1294.

(Rec.09/03/2018, Rev.28/05/2018, 18/09/2018, 18/10/2018, Ac. 13/11/2018) 